\title{
Autonomous thermography: towards the automatic detection and classification of building pathologies
}

\author{
by I. Garrido*, S. Lagüela** and P. Arias* \\ * Applied Geotechnologies Research Group, University of Vigo, Spain, ivgarrido; parias@uvigo.es \\ ** TIDOP Research Group, University of Salamanca, Spain, sulaguela@usal.es
}

\begin{abstract}
Infrared thermography (IRT) is widely used for the study of the composition of building envelopes and the detection of faults in the building materials with influence on their thermal behaviour such as thermal bridges and moistures. The automation of the interpretation of the thermal images is proposed as a solution to help in the identification of the pathologies, speeding up the inspection process as well as avoiding the loss of detection of existing pathologies, or their misinterpretation. Specifically, thermal bridges and moisture areas are detected and classified, and their contours are extracted through the temperature analysis of the thermal images.
\end{abstract}

\section{Introduction}

The detection, classification and contouring of building pathologies is necessary in order to take the corresponding maintenance measures. Among the most common and severe building pathologies are the thermal bridges and the moisture areas. The firsts are defined by the norm UNE-EN ISO 10211 as parts of the enclosure of a building where the thermal resistance presents significant changes due to geometrical changes (penetration in the enclosure of materials with different thermal conductivity, changes in thickness of the enclosure) [1]. The importance of these pathologies is shown by the existence of several IRT studies both for the analysis of thermal bridges, such as [2,3], and for the analysis of moistures $[4,5,6]$.

Regarding the previous examples of study, like all other IRT works, they deal with the problem that the resolution of the thermal images is lower than that of the visible images. Provided that the majority of image processing algorithms are developed for visible images, thermal images do not have the number of pixels necessary to present valid results. Moreover, the absolute temperature values of the thermal images are not enough to define each type of pathology (thermal bridge and moisture), since their characteristics, such as the temperature in the pathology and its unaltered surroundings, vary in each case.

Therefore, with the purpose to determine and classify both thermal bridges and moistures, IRT studies develop different algorithms for thermal images in order to take advantage of all the information contained in the images. In some researches, algorithms for image reconstruction and enhancement are applied, such as the use of the mathematical theory of the sampling Kantorovich operators [3]. Other studies apply statistical techniques in order to extract and separate different features present in the images and eliminate most of the existing noise, such as the Principal Component Thermography (PCT) method [4,5]. There are also studies that focus on the computation of different thermophysical properties and the comparison of pixel/temperature values in order to detect pathology contours, such as the linear thermal transmittance and the incidence factor calculation of each candidate to thermal bridge [2,3], the evaporative flux mapping of moisture areas on wall surfaces [6] and the temperature distribution analysis of each possible thermal bridge [2] and of the global image [3].

On the other hand, while the abnormal distribution of temperature in the areas of the thermal bridges and moistures with respect to the temperature distribution of their unaltered surroundings [7] allows for their detections, the different temperature evolution in their contours, makes possible the classification of each type of pathology, provided that the temperature difference between the interior and exterior surfaces of the building envelope analysed is significant (higher than $10^{\circ} \mathrm{C}$, as in a standard thermographic inspection [8]). This is because thermal bridges have a temperature change from their limits with the stable (non-pathology) areas (hereinafter, transition phase) more abrupt than the corresponding transition phase of the moistures, where the temperature value and the extent of the moisture/unaltered area depend on the water content.

Consequently, the thermal behaviour in the transition phase is taken as the first thermal criterion to apply in this paper, taking into account also the analysis of the temperature distribution of each thermal image under study in order to detect first, and then classify the type of pathology in an automated way. With this approach, the risk of missing and misunderstanding existing thermal bridges and moisture in buildings is minimized [2].

Furthermore, the extension of the pathologies is automatically calculated, being the final step of this work. For the acquisition of the contour of each pathology, PCT method and a second thermal criterion are applied. PCT is a technique proposed by Rajic in 2002 [9], which theory and adequate procedures according to the final objective are well known in the thermography field. Since it is based on a statistical approach for the processing of raw data [10], this tool has a lot of applications in active thermography, where it reduces a temporal sequence of thermal images from 3D data ( $x$, $y$ coordinates of the image, adding as third axis the total images of the sequence taken during the test) to different $2 \mathrm{D}$ thermal 
images (called Principal Components (PCs)), where each image describes partially the change of temperature value of each pixel along the time period. In this way, the first 2D thermal image (PC1) will describe the most part of the temperature variation of the pixel values of the object under study, decreasing the weight in the following images gradually, eliminating in the first 2D thermal image most of the existing noise. In this paper, PCT is applied to only one 2D thermal image in order to obtain various 1D temperature profiles (PCs). Therefore, using the first 1D temperature profile (PC1) in order to eliminate most of the existing noise, the detection of the boundaries of the areas of each pathology is possible.

This work is organized as follows: Section 2 describes the two thermal criteria and the particular application of the PCT method; Section 3 describes the methodology for the automatic detection, classification and extraction of geometrical boundaries of the pathologies under study; Section 4 presents the results of this methodology for thermal images belonging to different building envelopes. Last, Section 5 contains the conclusions reached after evaluation of the results.

\section{Assumptions}

This section details the two thermal criteria and the PCT technique used in this paper.

\subsection{First thermal criterion}

This criterion is based on the analysis of the temperature distribution of each thermal image under study, focusing on the transition phase between each pathology and their unaltered surroundings.

Generally speaking, pixel/temperature values belonging to a thermal bridge or a moisture are much lower/higher than those corresponding to unaltered zones of the building envelope, and they appear in a smaller proportion in number of pixels if the thermal image is taken with a global perspective of the building envelope under study. Therefore, the histogram of the temperature distribution of a thermal image of these characteristics can be assimilated to a bimodal distribution, with an increasing/decreasing temperature evolution between the thermal bridge/moisture contour and the corresponding stable zone of the unaltered surrounding (transition phase - highlighted between arrows in figure 1) and two peaks: the peak with the highest number of pixels representing the temperature area of the unaltered surrounding $(P 2$ in figure 1) and the lowest peak representing the temperature distribution of the thermal bridge/moisture (P1 in figure 1$)$.

Thus, the automatic detection of a thermal bridge/moisture in a thermal image is possible, checking if the temperature histogram is close to a bimodal distribution. This would be the basis of the first thermal criterion, completed with the analysis of the transition phase. By definition, the slope of the transition phase between the pathology and the corresponding unaltered surrounding (calculated between point $S$ and the middle point T2-P2 in figure 1) is higher in a thermal bridge than in a moisture, independent of the water content of the latter and as long as the temperature difference between the interior and exterior surfaces of the building envelope analysed is higher than $10^{\circ} \mathrm{C}$. So, by calculating the slope of the transition phase, automatic classification of the type of pathology in each thermal image can be done according to the value obtained.

The calculation of the slope of the transition phase is explained in Section 3.2.1.
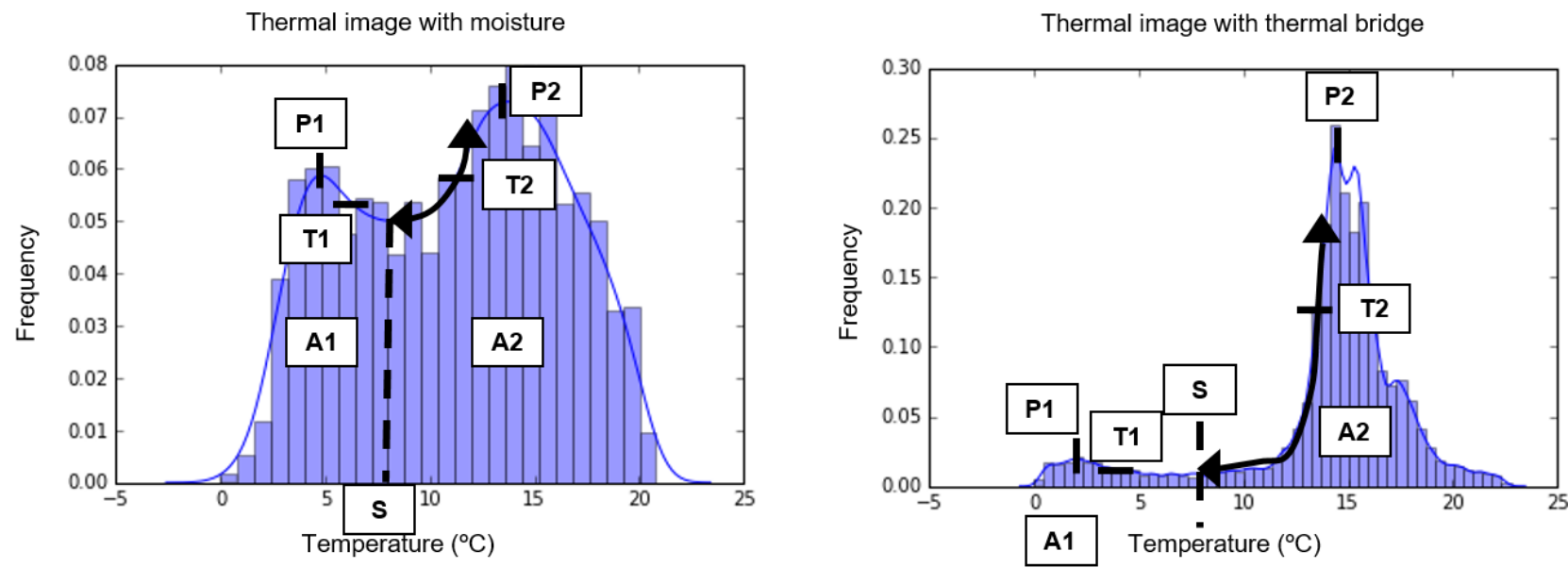

Fig. 1. Thermal image histograms with a moisture (left) and with a thermal bridge (right). Symbols $A 1, A 2, P 1$ and $P 2$ represent the pathology and unaltered areas, and their respective peaks. $S$ is the point that separates $A 1$ and $A 2$, and T1 and $T 2$ are the existing inflection points between $P 1$ and $P 2$. The arrow symbol represents the transition phase in each situation (important to observe the horizontal and vertical scale of the histograms). 


\subsection{PCT method and second thermal criterion}

The second phase is applied to extract the geometrical boundary of each pathology automatically, after their detection and classification with the first thermal criterion.

First, PCT technique is applied in different image blocks or windows. This way, the $2 \mathrm{D}$ temperature matrix is converted to $1 \mathrm{D}$ temperature profiles (PCs) in each window, where the most important is the first one (PC1), since it contains the most part of the variation among the pixel/temperature values along the columns of the window (horizontal profile) and eliminates most of the possible noise. Turning 90 degrees the image and performing the same procedure, the 1D temperature profile along the rows of each window (vertical profile) is obtained too.

Therefore, considering the inflection points (points where the values of a continuous mathematical function pass from one type of concavity to another) for each horizontal/vertical 1D temperature profile of each window, various lines are drawn on the image if the inflection points are prolonged in the perpendicular direction of their corresponding profiles. Areas between these drawn lines are assumed to be different areas with a maximum or minimum temperature peak.

Then, the second thermal criterion is applied in order to filter drawn lines which do not belong to the contour of the corresponding pathology. This consideration uses the first criterion as reference. Specifically, the pixels of each drawn line should have a temperature value close to the lowest extreme of the corresponding transition phase between the pathology and the unaltered zone ( $S$ point in figure 1$)$, since it represents the value that separates the areas of the pathology and of the unaltered zone. This last value will be the existing minimum peak between the two maximum peaks of the corresponding thermal image histogram (hereinafter, separating point).

As a result, line pixels whose temperature values are far from the value of the separating point, are discarded as possible parts of the contour of the pathology. Moreover, the mean temperature value should be higher to the separating point on one side of the line, and on the other side lower (sides corresponding to up/down in the case of horizontal lines and to left/right for vertical lines). So, lines with mean temperature values that do not fulfil the previous assumption are discarded as possible candidates too.

As an example, figure 2 represents the results of the application of the PCT and of the second thermal criterion in the thermal image with thermal bridge of figure 1.
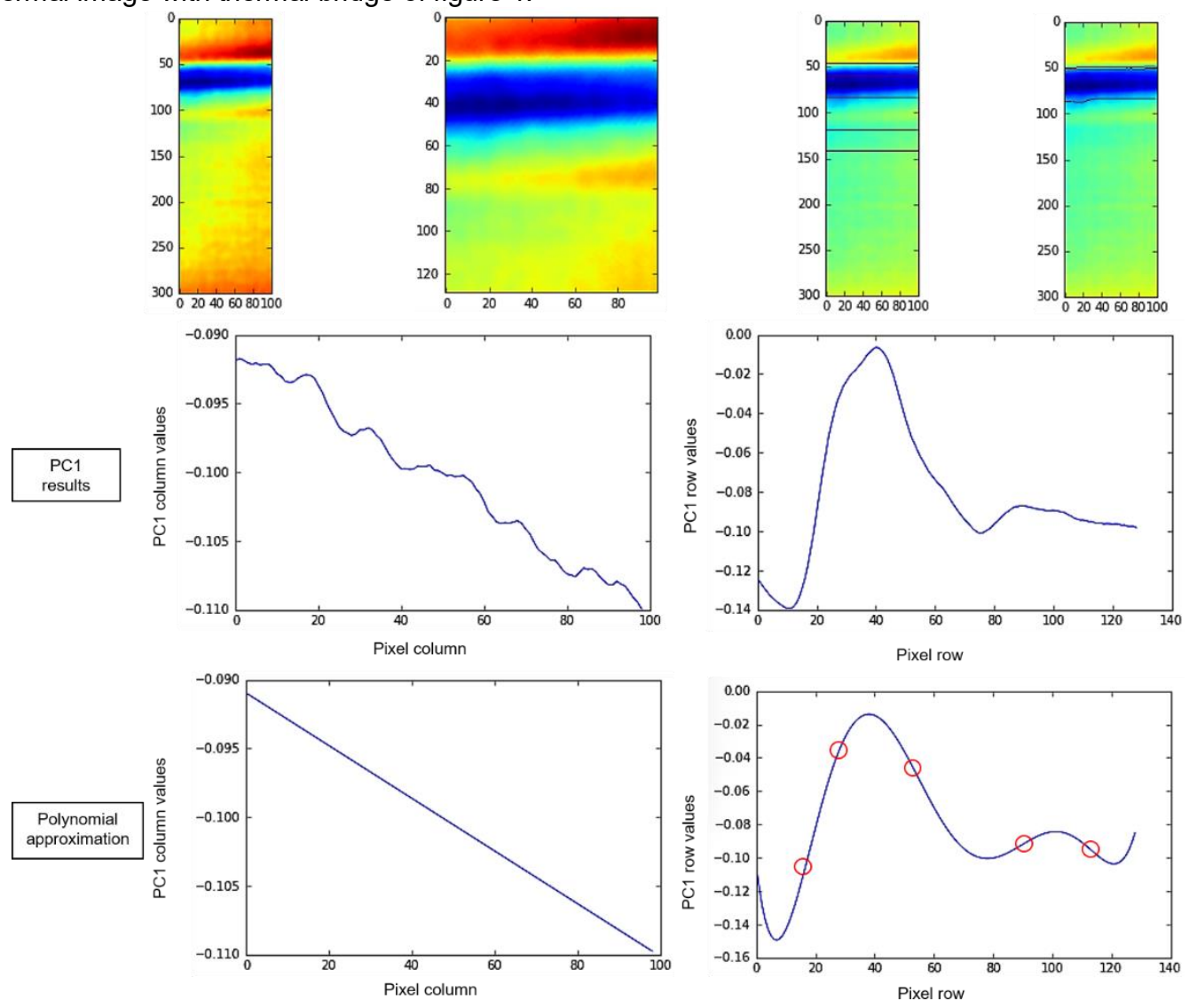

Fig. 2. In the first row and from left to right: original thermal image, window used to apply the PCT method, PCT results in the original image, and second thermal criterion results. In the second row and from left to right: graphs of 1D horizontal and $1 D$ vertical temperature profile of the PC1 results belonging to the PCT application. Third row and from left to right: the polynomial approximation of each temperature profile of the PC1 results, in order to obtain the existing inflection points (with a coefficient of determination higher than 0.95). Inflection points are drawn by red circles; while there are not inflection points in the horizontal profile, the vertical profile presents the following row pixels: 13, 32, 55, 90 and 115. 


\section{Methodology}

The methodology of this work is presented through the application of the first and second thermal criterion, and the PCT method to different thermal images. However, a pre-processing and a post-processing step are necessary.

\subsection{Preliminary steps (pre-processing)}

Having a 2D temperature matrix from a thermal image as input data, first an image processing technique is used to eliminate the majority of the noise existing in the image, keeping the contour of the thermal bridge/moisture. This technique is called bilateral filtering, and consists on the replacement of the pixel values of the image by their weighted average. This weighted average is based on a Gaussian distribution in space, which ensures that only the pixels close to the pixel under study are considered within its neighbourhood, and another Gaussian filter depending on the difference in pixels, which ensures that only pixels with a similar value to the pixel under study are considered as "blurring". With these filters, the edges of the thermal image are preserved, since the pixels in the contours of objects will have a great variation in value with respect to their surroundings [11].

Furthermore, a conversion of temperature values of the thermal image to 8-bit grey-level (0-255 pixel values) is required before the bilateral filtering, so that this technique works correctly. After bilateral filtering, the opposite process is applied, recovering the temperature values in the image, with an increase in the image contrast due to the following reasons: 1) the new interval of temperature values will have values with greater difference than the original interval, since the temperature interval is divided in 255 different values from the 8-bit grey-level format, a much higher number of values than the usual temperature interval in a thermal image 2) the original temperature interval is translated from [minimum original temperature value - maximum original temperature value] to $\left[0^{\circ} \mathrm{C}\right.$ - maximum original temperature value]. In this way, the detection, classification and extraction of geometrical boundaries of the pathologies are facilitated with the contrast enhancement. Figure 3 shows, again in the thermal image with thermal bridge of figure 1, the bilateral filtering and contrast enhancement results, respectively.
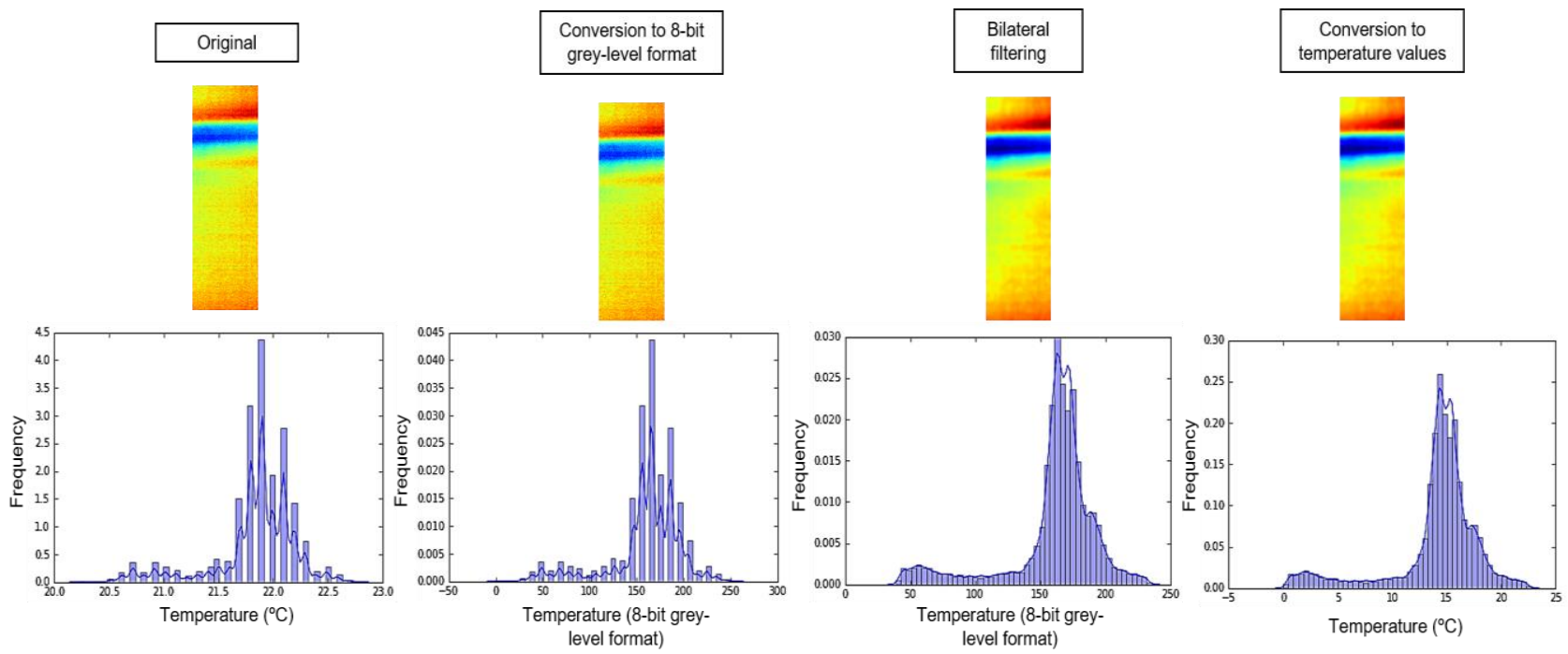

Fig. 3. Bilateral filtering and contrast enhancement results. Regarding "Conversion to 8-bit grey-level format", the thermal image histogram is stretched along the horizontal axis. In "Bilateral filtering", possible noise is the majority removed, and in "Conversion to temperature values", the pixels are converted back to temperature values. Therefore, the horizontal and vertical scales of the histogram show that the filtered image has its pixel values much more distributed than the original image, with lower number of pixels for each temperature value, but ten times more values; and with the filtered image with much less noise.

\subsection{Thermal criteria and PCT applications (core)}

\subsubsection{First thermal criterion: automatic pathology detection and classification}

In this step, the first thermal criterion is applied. In order to automate all the process, initially, a polynomial fit is adjusted to the shape of the thermal image histogram, with a high coefficient of determination in order to be as accurate as possible (minimum of 0.95 ). From the polynomial fit, the analysis of the maximum peaks of the polynomial equation allows to know if the thermal image presents a bimodal temperature distribution or not. For the case positive, there must be only two maximum peaks, each at one side of the middle of the polynomial. If this is the case, the automatic detection of a pathology is achieved. 
If a pathology is detected in the thermal image, the slope of the transition phase is calculated. For this, the previous polynomial equation is used again. In this case, the separating point, $S$ in figure 1 is considered as the minimum peak between the two maximum peaks of the polynomial. The highest extreme (point where the stable area of the unaltered surrounding would begin) is considered as the middle point between the inflection point $T 2$ and the maximum peak $P 2$ of figure 1, obtained with the corresponding polynomial equation. As a result, a slope value is calculated in each thermal image. The classification of the pathology is performed based on the threshold value of 0.015 [frequency/temperature $\left({ }^{\circ} \mathrm{C}\right)$ ], experimentally determined: higher slopes correspond to thermal bridges, while lower slopes come from moisture.

\subsubsection{PCT technique and second thermal criterion: automatic extraction of pathology boundaries}

In the second step of the core of the methodology, the PCT technique and the second thermal criterion are applied to all the thermal images where a pathology was detected and identified.

The windows used in order to divide the thermal image and to apply the PCT method, have been established as follows: 1) one window which rectangular area covers all the pixels with temperature values lower/higher than or equal to the temperature value of the highest extreme of the slope of the transition phase, adding a 21-pixel external radius on the area covered. The determination of lower or higher depends on the relative positions of the peaks P1 and P2 in the image histogram 2) four windows dividing the previous window into four equal rectangular areas.

After having the lines drawn in the thermal image (PCT results, always with a minimum coefficient of determination of 0.95 in the polynomial fit for the calculation of the inflection points), the second thermal criterion is applied to the pixels of each line. If the pixel value in the line is not between the corresponding inflection points $T 1$ and T2 (figure 1), it is discarded as candidate to be part of the contour of the pathology. Thus, the pixels of the lines which were not discarded so far, surrounded by a mean temperature higher than separating point on one side, and lower on the other side, are identified as the real contour pixels of the pathology under study, allowing the computation of the extension of the pathology detected in the thermal image analysed.

\subsection{Final steps (post-processing)}

The aim of the final steps is to connect automatically the pixels of the lines which have fulfilled the thermal criteria, forming a closed contour in each pathology. So, the following steps are implemented per image:

1) A dilation/erosion process is applied in the drawn pixels, to join non-continuous but neighbouring drawn pixels.

2) A "connecting components" function is applied (as in [12]), in order to group drawn pixels that represent a perfect line (pixels which have some side or vertex in common).

3) All the lines classified, are narrowed to 1D (vertical/horizontal lines with a width/height of 1 pixel respectively).

4) Lines with equal or less than 10 pixels are deleted (considered as lines with little importance) and overlapping lines are discarded, since in a specific zone of the contour of a pathology there may be two or more border lines overlapping each other.

5) Extremes of the final lines are joint by the equation of a line, since it is the minimum distance possible.

The results of each step are shown in figure 4, applied to the thermal image with a thermal bridge in figure 1.
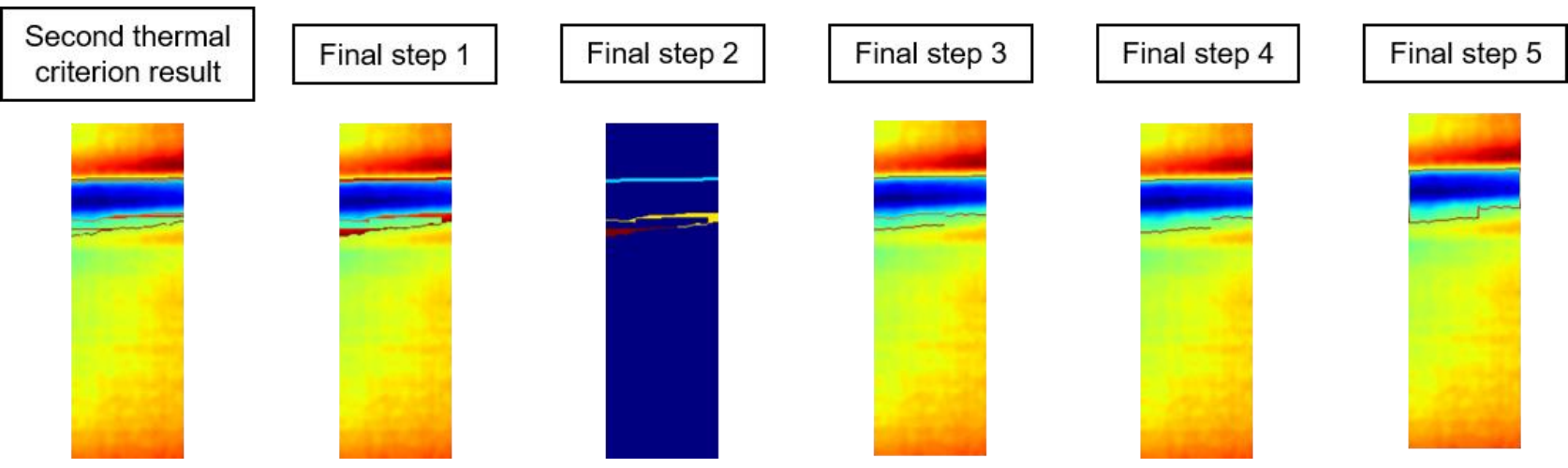

Fig. 4. Results of the sequence of the final steps. It is important to highlight that, in "Final step 2", the whole background of the image is coloured in blue, to facilitate the visualization of each line obtained.

\section{Results}

For the performance of the tests, 3 thermal images with thermal bridge areas and 3 thermal images with moisture areas were acquired in different exterior/interior residential building envelopes, located in the north of Spain and during the beginning of the night and in the spring season. The pathology, the nomenclature, the part of the building envelope and the environmental conditions of each image are shown in table 1. 
Table 1. Pathology, nomenclature, part of the envelope building and environmental conditions of each thermal image tested. Symbols $\mathrm{Ta}$ and $\mathrm{RH}$ are ambient temperature and relative humidity, respectively.

\begin{tabular}{|c|c|c|c|}
\hline $\begin{array}{l}\text { Pathology of the } \\
\text { thermal image }\end{array}$ & Nomenclature & $\begin{array}{l}\text { Part of the building envelope } \\
\text { that belongs to (surface } \\
\text { material) }\end{array}$ & $\begin{array}{l}\text { Environmental } \\
\text { conditions } \\
\text { (enclosure) }\end{array}$ \\
\hline \multirow{3}{*}{ Thermal bridge } & TB_1 & $\begin{array}{l}\text { Vertical intersection wall- } \\
\text { blind box (white plaster } \\
\text { surface) }\end{array}$ & $\begin{array}{l}\text { Ta: } 23^{\circ} \mathrm{C} \\
R H: 50 \% \\
\text { (living room) }\end{array}$ \\
\hline & TB_2 & $\begin{array}{l}\text { Horizontal intersection } \\
\text { ceiling-blind box (white } \\
\text { plaster surface) }\end{array}$ & $\begin{array}{l}\text { Ta: } 23^{\circ} \mathrm{C} \\
R H: 50 \% \\
\text { (bedroom) }\end{array}$ \\
\hline & TB_3 & $\begin{array}{l}\text { Horizontal intersection } \\
\text { balcony-wall (pink granite) }\end{array}$ & $\begin{array}{l}\text { Ta: } 12^{\circ} \mathrm{C} \\
R H: 80 \% \\
\text { (outdoor patio) }\end{array}$ \\
\hline \multirow{3}{*}{ Moisture } & M_1 & $\begin{array}{l}\text { Vertical intersection pillar- } \\
\text { wall (white plaster surface) }\end{array}$ & $\begin{array}{l}T a: 23^{\circ} \mathrm{C} \\
R H: 50 \% \\
\text { (living room) }\end{array}$ \\
\hline & M_2 & $\begin{array}{l}\text { Horizontal intersection } \\
\text { ceiling-wall (white plaster } \\
\text { surface) }\end{array}$ & $\begin{array}{l}\text { Ta: } 23^{\circ} \mathrm{C} \\
R H: 50 \% \\
\text { (living room) }\end{array}$ \\
\hline & M_3 & $\begin{array}{l}\text { Vertical intersection wall-wall } \\
\text { (white plaster surface) }\end{array}$ & $\begin{array}{l}\text { Ta: } 23^{\circ} \mathrm{C} \\
R H: 50 \% \\
\text { (bedroom) }\end{array}$ \\
\hline
\end{tabular}

The main specifications of the infrared (IR) camera used for data acquisition are described in table 2, where the corrections of emissivity and reflected temperature have been performed before each measurement, as well as ambient compensation.

Table 2. Specifications of the IR camera used during the tests.

\begin{tabular}{ll}
\hline & IR camera \\
\hline Model & NEC Thermo Tracer \\
& TH9260 \\
Uncooled focal plane & array $(\mu$ bolometer $)$ \\
Sensor type & $640(\mathrm{H}) \times 480(\mathrm{~V})$ pixels \\
Thermal image & $0.1^{\circ} \mathrm{C}$ \\
pixels & $\pm 2^{\circ} \mathrm{C}$ or $\pm 2 \%$ \\
Resolution & $8-14 \mu \mathrm{m}$ \\
Accuracy & \\
Spectral ranges & \\
\hline
\end{tabular}

Figure 5 shows the visible and thermal images obtained for each test, in RGB and with their temperature scales in ${ }^{\circ} \mathrm{C}$. Figure 6 represents the results of each step of the methodology presented in this paper, for each thermal image analysed. It is important to highlight that, in each original thermal image, only the region of interest (ROI) has been used to avoid having different types of pathologies in the same image, and that the steps of the methodology shown are: preprocessing results (Step 1), PCT results (Step 2), second thermal criterion results (Step 3) and post-processing/final results (Step 4).
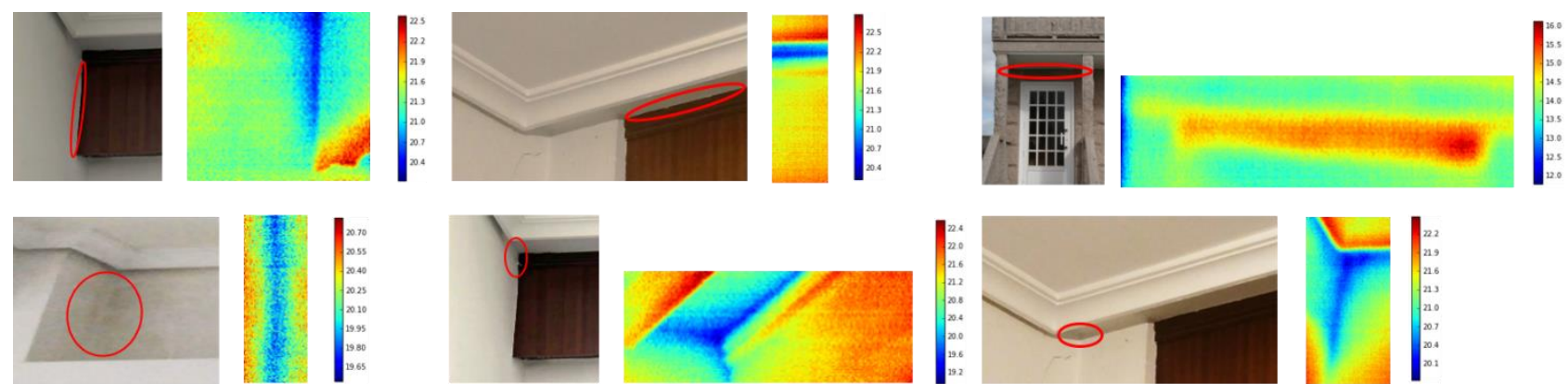

Fig. 5. Visible and thermal image of TB 1, TB 2, TB 3, M 1, M 2 and $M$ 3, where the ROI is highlighted with a red circle in the visible image. The temperature scale $\left({ }^{\circ} \mathrm{C}\right)$ is shown at the right of each thermal image. 


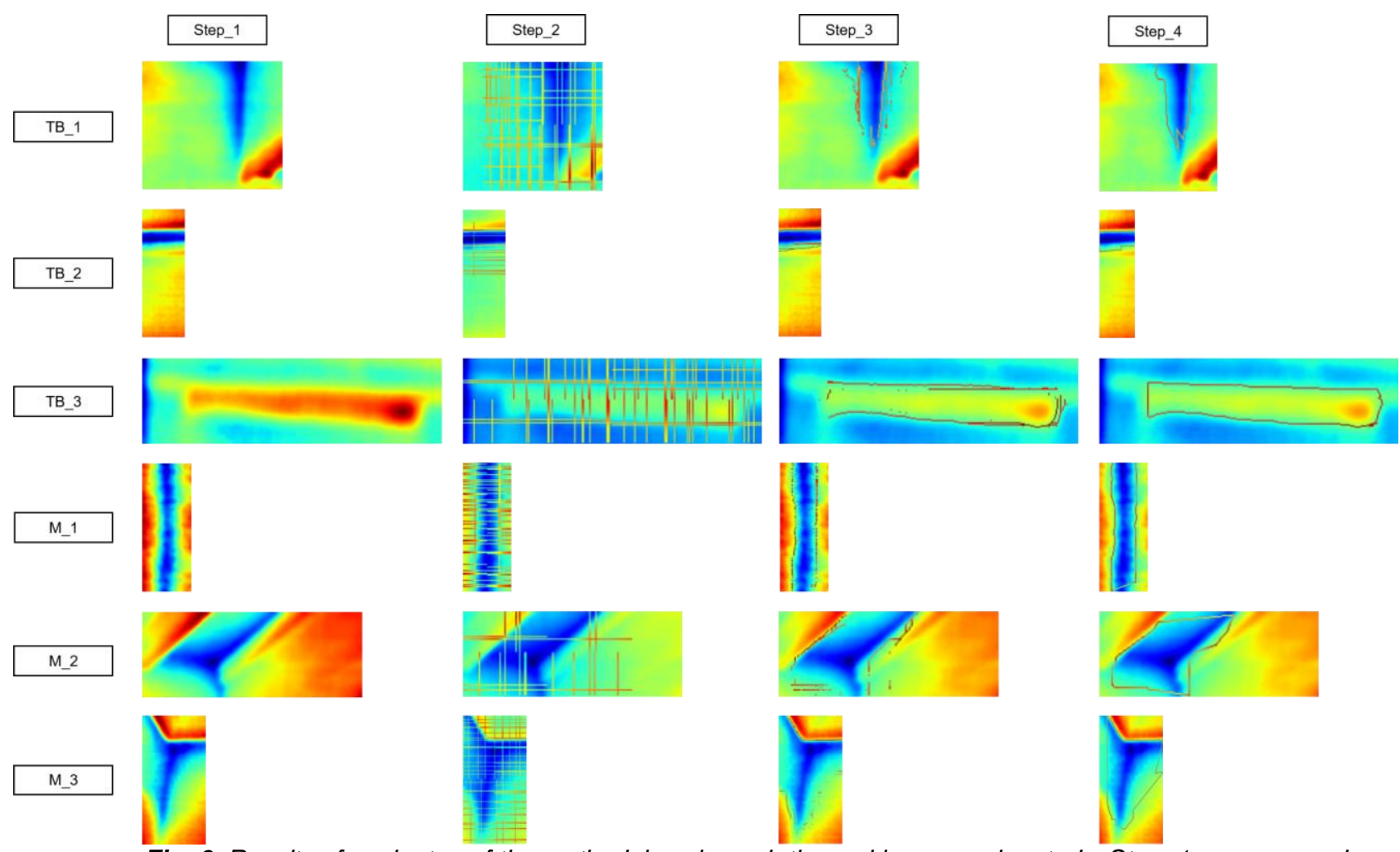

Fig. 6. Results of each step of the methodology in each thermal image under study. Step_1: pre-processing results, Step_2: PCT results, Step_3: second thermal criterion results and Step_4: post-processing/final results.

With the purpose of evaluating the accuracy of this work, various performance metrics are computed. To that end, figure 7 shows the real and calculated extensions of the existing thermal bridges and moistures in each thermal image analysed.

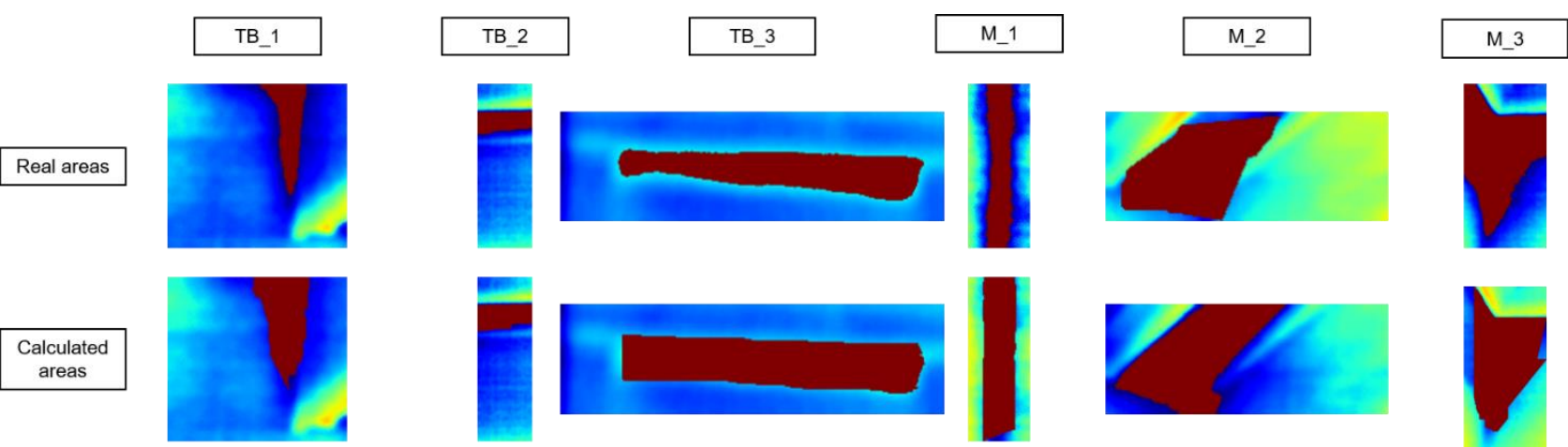

Fig. 7. Real and calculated areas of the existing thermal bridges and moistures in the thermal images tested.

And the performance metrics used are the following:

Precision $=\frac{T P}{T P+F P}$

Recall $=\frac{T P}{T P+F N}$

$F-$ score $=2 * \frac{\text { Precision } * \text { Recall }}{\text { Precision }+ \text { Recall }}$

where $T P$ is the number of true positives or number of coincident pixels between the pixels of the calculated area and the pixels of the corresponding real area, FP is the number of false positives or number of pixels present in the calculated area but not included in the real area and $F N$ is the number of false negatives or number of pixels of the real area which were not included in the corresponding calculated area. 
Therefore, table 3 shows the results of precision, recall and F-score performance metrics for each thermal image tested. In addition, table 4 represents the slope values of the transition phase of each thermal image analysed, and the average and standard deviation values, considering all the thermal bridges and all the moistures, respectively, corroborating that the threshold value of 0.015 [frequency/temperature $\left({ }^{\circ} \mathrm{C}\right)$ ], would be adequate for the automatic classification of the pathology in thermal bridge or moisture.

Table 3. Precision, recall and F-score in each thermal image tested.

\begin{tabular}{llll}
\hline Reference & Precision (\%) & Recall (\%) & F-score (\%) \\
\hline TB1 & 60 & 97 & 74 \\
TB2 & 82 & 100 & 90 \\
TB3 & 71 & 99 & 83 \\
M1 & 70 & 97 & 81 \\
M2 & 84 & 89 & 86 \\
M3 & 91 & 82 & 86 \\
\hline
\end{tabular}

Table 4. Slope values of the transition phase of each thermal image.

\begin{tabular}{llll}
\hline Reference & $\begin{array}{l}\text { Slope_values } \\
\text { (frequency/temperature }\left({ }^{\circ} \mathrm{C}\right) \text { ) }\end{array}$ & Average value & Standard deviation \\
\hline TB1 & 0.02 & 0.036 & 0.011 \\
TB2 & 0.047 & & \\
TB3 & 0.04 & & 0.001 \\
\hline M1 & 0.005 & 0.007 & \\
M2 & 0.008 & & \\
M3 & 0.008 & & \\
\hline
\end{tabular}

In order to obtain a more global vision of the results of table 3 , table 5 shows the average and standard deviation values of each performance metric, taking into account only thermal images with thermal bridges, only images with moistures and all the images.

Table 5. Average and standard deviation values of each performance metric, taking into account only thermal images with thermal bridges, only images with moistures and all the images.

\begin{tabular}{lllll}
\hline & & Precision (\%) & Recall (\%) & F-score (\%) \\
\hline \multirow{2}{*}{ Thermal bridges } & Average & 71 & 99 & 82 \\
& Standard deviation & 9 & 1 & 7 \\
\hline \multirow{2}{*}{ Moistures } & Average & 82 & 89 & 85 \\
& Standard deviation & 9 & 6 & 2 \\
\hline \multirow{2}{*}{ Global } & Average & 76 & 94 & 83 \\
& Standard deviation & 10 & 7 & 5 \\
\hline
\end{tabular}

In light of the results shown in tables 3 and 5 , a value equal to or higher than $70 \%$ is obtained, in most cases, in all the parameters, being generally higher values in recall than in precision.

On the other hand, this methodology detects better real areas of thermal bridges than real areas of moistures, being the recall results of $99 \% \pm 1 \%$ in the thermal bridges and $89 \% \pm 6 \%$ in the moistures tested. However, this procedure detects with more overestimated defects in thermal images with thermal bridges than with moistures, being the precision results of $71 \% \pm 9 \%$ in the thermal bridges and $82 \% \pm 9 \%$ in the moistures under study. This may be due to the more abrupt temperature jump of the thermal bridges with respect to their unaltered surroundings in front of moistures. In other words, since the areas of the thermal bridges are more "confined", their real areas will be easier to extract, but, at the same time, it is more difficult to determine the pixels that are within the pathology area without overestimated defects due to the higher slope of the temperature evolution in the proximities of their contours. In contrast, areas with moistures are not so well defined and, therefore, it is more difficult to extract their real areas but, at the same time, it is easier to identify the pixels that are within their areas without overestimated defects due to the lower slope of the temperature evolution in the proximities of their contours.

In any case, the variation of the percentage values between thermal bridges and moistures are minimal in the performance metrics results, where the F-score results, which considers equal weight for the previous parameters, are practically the same with $82 \% \pm 7 \%$ and with $85 \% \pm 2 \%$ for thermal images with thermal bridges and with moistures, respectively.

Because of the detection of the pathologies in the thermal images is done with the first thermal criterion, the recall parameter is more important than the precision parameter in F-score. In addition, in the automatic extraction of the contours 
of the pathologies, it is preferable to exceed than to underestimate the calculated areas for the necessary maintenance measures. Thus, a new $F$-score is defined using Eq. (4), being $x$ and $y$ the new weights for recalland precision, respectively:

$F-$ score $^{\prime}=\frac{\text { Precision } * \text { Recall }}{x * \text { Precision }+y * \text { Recall }}$

The new F-score increases for thermal bridges, for moistures and globally given the following weights in precision/recall (\%) (x/y in brackets): 40/60 (60/40), 30/70 (70/30), 20/80 (80/20) and 10/90 (90/10) (table 6):

Table 6. Average and standard deviation values in the new F-score parameter (precision/recall weights (\%): (1) 50/50, (2) 40/60, (3) 30/70, (4) 20/80 and (5) 10/90), taking into account only thermal images with thermal bridges, only

\begin{tabular}{lllllll}
\multicolumn{7}{c}{ images with moistures and all the images). } \\
\hline \multirow{2}{*}{$\begin{array}{l}\text { Thermal } \\
\text { bridges } \\
(50 / 50)\end{array}$} & $\begin{array}{l}\text { Average } \\
\text { Standard } \\
\text { deviation }\end{array}$ & 82 & $\begin{array}{l}\text { F-score' (\%) } \\
(40 / 60)\end{array}$ & $\begin{array}{l}\text { F-score' (\%) } \\
(30 / 70)\end{array}$ & $\begin{array}{l}\text { F-score' (\%) } \\
(20 / 80)\end{array}$ & $\begin{array}{l}\text { F-score' (\%) } \\
(10 / 90)\end{array}$ \\
\hline \multirow{2}{*}{ Moistures } & Average & 85 & 6 & 88 & 91 & 95 \\
& $\begin{array}{l}\text { Standard } \\
\text { deviation }\end{array}$ & 2 & 85 & 5 & 4 & 3 \\
\hline \multirow{2}{*}{ Global } & $\begin{array}{l}\text { Average } \\
\text { Standard }\end{array}$ & 83 & 1 & 86 & 87 & 88 \\
& $\begin{array}{l}\text { Standion } \\
\text { deviation }\end{array}$ & 85 & 4 & 87 & 3 & 4 \\
\hline
\end{tabular}

\section{Conclusions}

This paper presents a methodology for the automatic detection and classification of building pathologies, including the extraction of their geometrical boundaries. Specifically, two of the most important and severe pathologies are studied through the temperature analysis of the thermal images which contain these pathologies: thermal bridges and moistures. The study is based on two thermal criteria and the PCT method.

After the application of an image processing technique, in order to remove noise while keeping the edges intact, and the improvement of the contrast on the thermal images analysed, as a pre-processing step, the first thermal criterion is applied. With this criterion, the automatic detection of a possible thermal bridge/moisture in a thermal image is achieved, analysing if the image presents a bimodal temperature distribution. In the case of positive detection, the automatic classification of the type of pathology is also obtained. For this, the slope value of the temperature evolution is calculated from the minimum peak (separating point) between the two maximum peaks of the bimodal distribution of the image analysed to near the maximum peak (middle point between T2 and P2 of figure 1), representing the unaltered zone in the thermal image. The value of the slope allows to distinguish between a thermal bridge and a moisture, since the slope of the temperature evolution from the contour of a pathology to the stable zone of its unaltered surrounding (transition phase) is higher in the thermal bridges due to their confined nature.

The PCT technique and the second thermal criterion are used for the automatic extraction of the contours of the pathologies detected in the thermal images. PCT is applied in different image windows, in order to draw various lines in the thermal image which represent divisions of different areas with a maximum or a minimum temperature peak, including the area of the pathology. With the purpose of eliminating false divisions/drawn lines, the second thermal criterion is applied, continuing to analyse the bimodal distribution of the corresponding thermal image. Therefore, with the application of a post-processing step in order to connect automatically the pixels of the lines which have fulfilled the thermal criteria, the limits of the pathologies are obtained.

Various performance metrics are performed for the evaluation of the methodology, based on the comparison of the calculated areas with respect to real areas; in particular, precision, recall and F-score parameters are computed. It is important to highlight that in all the tests, the assumption of bimodal temperature distribution in each thermal image has been fulfilled, with a $71 \% \pm 9 \%$ in the thermal bridges and with a $82 \% \pm 9 \%$ in the moistures tested regarding precision result; with a $99 \% \pm 1 \%$ in the thermal bridges and with a $89 \% \pm 6 \%$ in the moistures tested regarding recall results and with a $82 \% \pm 7 \%$ in the thermal bridges and with a $85 \% \pm 2 \%$ in the moistures tested regarding $F$-score results. So it can be said that all the performance metrics are higher than $70 \%$, and that the methodology detects better real areas of thermal bridges than real areas of moistures (better recall results) but with more overestimated defects (worse precision results), probably due to the fact that thermal bridge areas are more confined and that the temperature jumps in the proximities of their contours are higher. However, the percentage values between thermal bridges and moistures are very similar, specially the F-score parameter, which considers equal weight with regard to the precision and recall parameters. Furthermore, since the detection of the pathologies in the thermal images is done with the first thermal criterion, the recall parameter is more important than the precision parameter in F-score. Thus, the new F-score increases gradually by changing the precision (towards lower value)/recall (towards higher value) weights, resulting in the following values considering a precision/recall weight percentage of $10 \% / 90 \%$ : $95 \% \pm 3 \%, 88 \% \pm 4 \%$ and $91 \% \pm 5 \%$, only for thermal bridges, only for moistures, and for both, respectively. 
In conclusion, this paper implies a new step for the automation of the IRT inspections in buildings with respect to the presence of thermal bridges and moistures, with results that would be useful for the calculation of the areas of the pathologies from the knowledge of the calibration parameters of the IR camera used in each study, the obtaining of several thermophysical properties characterizing each pathology analysed, such as the thermal transmittance, and the calculation of heat transfer through these calculated areas. In addition, future criteria and techniques can be added for the improvement of this methodology, such as using different image windows to apply the PCT technique in the thermal image under study or developing new criteria for the automatic detection, classification and extraction of contours in a thermal image with thermal bridges and moistures. Future researches will deal with these concepts, in addition to testing other construction materials.

\section{REFERENCES}

[1] UNE-EN ISO 10211, Puentes térmicos en edificación. Flujos de calor y temperaturas superficiales, Cálculos detallados, 2012.

[2] Garrido I., Lagüela S., Arias P., Balado J., Thermal-based analysis for the automatic detection and characterization of thermal bridges in buildings, Energy Build. 158, pp. 1358-1367, 2018.

[3] Baldinelli G., Bianchi F., Rotili A., et al., A model for the improvement of thermal bridges quantitative assessment by infrared thermography, Appl. Energy. 211, pp. 854-864, 2018.

[4] Edis E., Flores-Colen I., de Brito J., Quasi-quantitative infrared thermographic detection of moisture variation in facades with adhered ceramic cladding using principal component analysis, Build. Environ. 94, pp. 97-108, 2015.

[5] Sfarra S., Ibarra-Castanedo C., Tortora M., et al., Diagnostics of wall paintings: A smart and reliable approach, J. Cult. Herit. 18, pp. 229-241, 2016.

[6] Grinzato E., Ludwig N., Cadelano G., et al., Infrared Thermography for Moisture Detection: A Laboratory Study and In-situ Test, Society for Nondestructive Testing, 2011.

[7] Bagavathiappan S., Lahiri B., Saravanan T., et al., Infrared thermography for condition monitoring - A review, Infrared Phys. Technol., 60, pp. 35-55, 2013.

[8] López-Fernández L., Lagüela S., González-Aguilera D., et al., Thermographic and mobile indoor mapping for the computation of energy losses in buildings. Indoor and Built Environment, 26, pp. 771-784, 2017.

[9] Rajic N., Principal component thermography for flaw contrast enhancement and flaw depth characterisation in composite structures, Compos. Struct. 58, pp. 521-528, 2002.

[10] Gavrilov D., Maev R., Extraction of Independent Structural Images for Principal Component Thermography, Appl. Sci. 8, pp. 459, 2018.

[11] Mordvintsev A., Rahman A., OpenCV-Python Tutorials Documentation, 2017.

[12] Balado J., Díaz-Vilariño L., Arias P., et al., Automatic building accessibility diagnosis from point clouds, Autom. Constr. 82, pp. 103-111, 2017. 